\title{
Emotional triggering and low socio-economic status as determinants of depression following acute coronary syndrome
}

\author{
A. Steptoe ${ }^{1 *}$, G. J. Molloy ${ }^{2}$, N. Messerly-Bürgy ${ }^{3}$, A. Wikman ${ }^{1}$, G. Randall ${ }^{1}$, L. Perkins-Porras ${ }^{4}$ \\ and J. C. Kaski ${ }^{5}$ \\ ${ }^{1}$ Department of Epidemiology and Public Health, University College London, London, UK \\ ${ }^{2}$ Department of Psychology, University of Stirling, Stirling, UK \\ ${ }^{3}$ Department of Clinical Psychology and Psychotherapy, University of Bern, Bern, Switzerland \\ ${ }^{4}$ Division of Community Health Sciences, and ${ }^{5}$ Division of Cardiac and Vascular Sciences, St. George's, University of London, London, UK
}

Background. The determinants of depression following acute coronary syndrome (ACS) are poorly understood. Triggering of ACS by emotional stress and low socio-economic status (SES) are predictors of adverse outcomes. We therefore investigated whether emotional triggering and low SES predict depression and anxiety following ACS.

\begin{abstract}
Method. This prospective observational clinical cohort study involved 298 patients with clinically verified ACS. Emotional stress was assessed for the $2 \mathrm{~h}$ before symptom onset and compared with the equivalent period $24 \mathrm{~h}$ earlier using case-crossover methods. SES was defined by household income and education. Depression was measured with the Beck Depression Inventory and the Hamilton Rating Scale for Depression and anxiety with the Hospital Anxiety and Depression Scale 3 weeks after ACS and again at 6 and 12 months. Age, gender, ethnicity, marital status, the Global Registry of Acute Coronary Events risk score, duration of hospital stay and history of depression were included as covariates.
\end{abstract}

Results. Emotional stress during the 2-h hazard period was associated with increased risk of ACS (odds ratio 1.88, $95 \%$ confidence interval 1.01-3.61). Both low income and emotional triggering predicted depression and anxiety at 3 weeks and 6/12 months independently of covariates. The two factors interacted, with the greatest depression and anxiety in lower income patients who experienced acute emotional stress. Education was not related to depression.

Conclusions. Patients who experience acute emotional stress during their ACS and are lower SES as defined by current affluence and access to resources are particularly vulnerable to subsequent depression and anxiety.

Received 5 August 2010; Revised 25 November 2010; Accepted 2 December 2010; First published online 7 January 2011

Key words: Acute coronary syndrome, anxiety, depression, emotional triggering, physical exertion, socio-economic status.

\section{Introduction}

Depressive symptoms in the weeks following a nonfatal acute coronary syndrome (ACS) are common, with $15-20 \%$ patients experiencing severe depression and a further $20 \%$ reporting moderate symptoms (Thombs et al. 2006; Lichtman et al. 2008). Depression in the first months after an ACS predicts recurrent coronary events, mortality from coronary heart disease (CHD), poor quality of life, greater healthcare utilization and failure to return to work (Nicholson et al. 2006; Bhattacharyya et al. 2007; Kurdyak et al.

* Address for correspondence: Dr A. Steptoe, Department of Epidemiology and Public Health, University College London, 1-19 Torrington Place, London WC1E 6BT, UK.

(Email: a.steptoe@ucl.ac.uk)
2008). These associations are largely independent of markers of disease severity and cardiovascular risk profiles (Kronish et al. 2009). Investigation of the mechanisms relating depression with future cardiovascular morbidity is an active field of research, with candidates including biological processes such as autonomic, inflammatory, neuroendocrine and endothelial dysfunction, together with deficits in adherence to medication and life-style advice (Grippo \& Johnson, 2009; de Jonge et al. 2010).

The determinants of depression following ACS are not well understood. History of depressive illness is relevant, but there is accumulating evidence that new onset depression in individuals without a positive history may be particularly cardiotoxic (de Jonge et al. 2006; Dickens et al. 2008; Parker et al. 2008; Carney et al. 2009). Clinical depression and depressed mood in 
the general population are inversely associated with socio-economic status (SES) (Lorant et al. 2003). Lower SES also predicts adverse outcomes after ACS, including more frequent rehospitalization, impaired functional status and mortality (Ickovics et al. 1997; Alter et al. 2006a). The impact of SES on the development of depression following ACS has been inconsistent. Education shows mixed associations with depression incidence in cardiac patients (FrasureSmith et al. 1993; Martens et al. 2008; Carney et al. 2009), but may be a weak indicator of socio-economic circumstances in this population since most patients will have completed their education 30-40 years before developing CHD. It may be more appropriate to study contemporaneous measures of SES, such as income or socio-economic deprivation. One aim of this study was therefore to compare income and education as predictors of depressed mood following ACS. We hypothesized that depression 3 weeks after ACS would be associated with income but not education independently of clinical factors, disease severity and history of clinical depression.

Another relevant factor may be the emotional experience of patients in the period immediately preceding onset of acute ACS symptoms. A proportion of ACS is thought to be stimulated by triggers, defined as short-term stimuli or activities that precipitate the acute physiological or pathophysiological changes that initiate severe cardiac dysfunction (Steptoe \& Brydon, 2009). Triggers include physical exertion, heat and cold stress, infection and emotional stimuli such as acute anger, sadness and stress (Strike \& Steptoe, 2005; Kloner, 2006; Warren-Gash et al. 2009). Some studies have involved population-based investigations of events such as earthquakes and sports matches, but most research has been based on individual retrospective reports, analysed using case-crossover methods (Maclure \& Mittleman, 2000). The long-term consequences of emotional triggering have not been studied extensively, but a recent analysis from our group found that emotional triggering was associated with elevated anxiety 3 years after an ACS (Bhattacharyya et al. 2010). Notably, the biological mechanisms thought to be responsible for triggering, involving arterial plaque disruption coupled with autonomic dysfunction, inflammation, prothrombotic responses and neuroendocrine activation (Steptoe \& Brydon, 2009), are similar to those implicated in depression following ACS. The second aim of this study was to measure the incidence of triggering of ACS by emotional stress and physical exertion and to test associations with subsequent depressive symptoms.

We also tested whether lower SES and emotional triggering act synergistically in predicting depressive symptoms following ACS. Triggering of ACS by negative emotions appears to be more common among lower SES individuals (Mittleman et al. 1997; Strike et al. 2006b). Lower SES increases vulnerability to depressive symptoms following stressful events in general (Kendler et al. 2002), so may have an analogous impact on the occurrence of depression following emotional triggering of ACS.

\section{Method}

\section{Study population}

Participants were 298 patients admitted with ACS to St. George's Hospital in South London between June 2007 and October 2008. Patients were included if they had a diagnosis of ACS based on the presence of chest pain plus verification by diagnostic electrocardiographic (ECG) changes, troponin $\mathrm{T}$ or troponin $I \geqslant 99$ th percentile of the upper reference limit and/or a creatine kinase measurement more than twice the upper range of normal for the measuring laboratory (Thygesen et al. 2007). Additional inclusion criteria were age of $\geqslant 18$ years, absence of co-morbid conditions that might influence either symptom presentation or mood, other conditions that might cause troponin positivity and ability to complete interviews and questionnaires in English. The study was approved by the Wandsworth Research Ethics Committee and written consent was obtained.

In-hospital data were collected between June 2007 and October 2008. On the days that recruitment was conducted, 666 potentially eligible patients were admitted. Of these, 125 (19\%) had been discharged or transferred to a different hospital before the patient could be recruited into the study, 90 (14\%) were too clinically fragile (e.g. critical ischaemia, ventricular tachyarrhythmia) to take part, 58 patients (9\%) declined to participate, 27 (4\%) could not speak English, $23(3 \%)$ were in confusional states, seven patients $(1 \%)$ died in hospital and a further $38(6 \%)$ were excluded for other reasons. Patients who were not included in the study were older on average than those who did take part (mean $66.7 \pm 14.0$ v. $60.2 \pm 11.6$ years, $p<0.001)$ and were more likely to be female $(32.0 \% v$. $16.1 \%, p<0.001)$.

\section{Assessment of acute triggers}

Patients were interviewed in hospital an average of $2.41 \pm 1.6$ days after admission with a structured triggering interview based on the Determinants of Myocardial Infarction Onset study and other investigations (Mittleman et al. 1995; Moller et al. 2005; Strike et al. 2006b). Patients were questioned about the circumstances surrounding the onset of acute 
symptoms. Based on previous evidence, we specifically focused on activities and emotional states during a 2-h period before symptom onset. Vigorous physical exertion was defined as activity of at least six metabolic equivalents, as used in earlier studies (Mittleman et al. 1993; Willich et al. 1993). Anger, stress and acute depression or sadness during each hour before symptom onset were assessed on a set of rating scales (ranging from $0=$ none to $4=$ extreme) and the reasons for these emotional states were probed. Because the experience of stress, depression and anger during the hazard period overlapped, we based the analysis on combined emotional triggers, rather than the three moods separately. Patients were asked identical questions about the same period of the day $24 \mathrm{~h}$ earlier for the pair-matched, case-crossover analysis.

\section{Socio-economic measures}

SES was assessed using income and education. Household income was collected in five categories, reduced to three for the purposes of analysis: $<£ 20000$ per annum; $£ 20000-£ 40000$ per annum; $>£ 40000$ (equivalent to around $<\$ 30000, \$ 30000-60000$ and $>\$ 60000$, respectively). Education was classified into three groups: primary (basic qualifications); secondary (high school); college/university education.

\section{Clinical measures}

Admission ECGs were reviewed and scrutinized for presentation as ST-elevation myocardial infarction (STEMI) or non-STEMI/unstable angina. We computed the composite risk index based on the algorithm developed in the Global Registry of Acute Coronary Events (GRACE) study (Eagle et al. 2004). This uses nine criteria (age, history of congestive heart failure, history of myocardial infarction (MI), systolic blood pressure and heart rate on admission, ST segment depression, initial serum creatinine, elevated cardiac enzymes and in-hospital percutaneous coronary intervention) to define risk of 6-month post-discharge death applicable to all types of ACS. The GRACE index was selected because it performs effectively across the SES spectrum (Alter et al. 2006b). The number of days spent in hospital was also recorded.

\section{Assessment of depression and anxiety}

Patients were interviewed in their own homes an average 21.6 days following the ACS, when the Beck Depression Inventory (BDI) and anxiety scale from the Hospital Anxiety and Depression Scale (HADS) were completed (Zigmond \& Snaith, 1983, Beck et al. 1988). The BDI consists of 21 items rated on a scale of $0-3$, so maximum scores can range from $0-63$. Patients were also categorized on the basis of giving BDI scores $\geqslant 10$. Anxiety scores could range from $0-21$ with higher values indicating greater anxiety and individuals were also categorized with anxiety scores $\geqslant 8$. The Cronbach $\alpha$ in this sample was 0.86 and 0.88 respectively. Patients were also administered the Depression Interview and Structured Hamilton (DISH) developed for the Enhancing Recovery in Coronary Heart Disease study, from which a 17-item Hamilton Rating Scale for Depression (HAMD) score was derived (Freedland et al. 2002). As part of the DISH, participants were also questioned about their psychiatric history. Lifetime history of clinical depression was defined as having been diagnosed with depressive disorder and/or taking antidepressant treatments in the past, while pre-ACS depression was defined as a clinical diagnosis within the last 12 months and/or current antidepressive medication.

Assessments of depression and anxiety with the BDI and HADS were repeated at 6 and 12 months following semi-structured telephone interviews with patients by trained interviewers.

\section{Other measures}

Habitual physical activity in the months before ACS onset was assessed by asking patients on how many days per week they were sufficiently vigorously active to be out of breath. Chronic stress in the 6 months before ACS was measured with a series of rating scales (from $0=$ none to $4=$ extreme) asking about stress in five domains: relationship with partner; relationships in the family; work; other illnesses; financial issues. A total score for stress in the past 6 months was derived. The occurrence of major adverse cardiac events (further ACS, cardiac readmission, surgery or death from cardiac causes) between ACS and follow-up was included as a covariate in the regression analyses on long-term emotional adaptation.

\section{Statistical analysis}

Triggering by emotional stress and physical exertion was analysed using the case-crossover approach as detailed by Maclure \& Mittleman (2000). Onset of ACS was defined as the time at which the symptoms that led to admission began. Analyses were carried out separately of emotional stress and physical exertion. Risk of exposure to emotional stress or physical exertion during the 2-h hazard period before ACS onset was calculated relative to the same 2-h period that occurred $24 \mathrm{~h}$ earlier (pair-matched analysis). The pair-matched case-crossover design is analogous to a matched-pair case-control design, but with each individual serving as his or her own control. Therefore, 
frequencies of discordant and concordant exposure in the control and hazard periods are used to derive an odds ratio (OR) with $95 \%$ confidence intervals (CI).

The characteristics of participants in the different income categories were compared using $\chi^{2}$ tests for categorical and analysis of variance for continuous variables. The relationship between SES (income or education), triggering by emotional stress or physical exertion, and depression and anxiety scores at 3 weeks and 6 or 12 months was analysed with analysis of covariance, with SES and trigger category as betweenperson variables and age, gender, ethnicity, marital status, GRACE risk score, days in hospital (log transformed) and depressive history as covariates. Logistic regressions on BDI depression $\geqslant 10$ and HADS anxiety $\geqslant 8$ were also carried out, involving an interaction term between SES and triggering and the same covariates. In the analyses of longer-term emotional status, the occurrence of major cardiac events in the intervening period was included as an additional covariate. Results are presented as adjusted means with standard deviations and adjusted ORs with $95 \%$ CI.

\section{Attrition analysis}

Adequate income data were obtained from 279 of the 298 patients and information about emotional experience during the hazard and control periods was obtained from 273 patients (258 provided data on both measures). The remainder were not included in the analysis, because they refused or were unable to provide data on income or were asleep in the 2-h hazard period before symptom onset or during the corresponding control period $24 \mathrm{~h}$ earlier. There were no significant differences between the patients who did and did not provide income and acute emotional experience data on any factors. Altogether, 226 patients were assessed for depression 3 weeks after admission (76\%). In total, 40 patients $(13 \%)$ declined to participate in the second phase of the study, $12(4 \%)$ patients were excluded because of extreme ill health (patient too unwell to complete follow-up interview, readmission to hospital, memory problems) and 21 (7\%) patients could not be re-contacted despite repeated efforts by telephone and mail. Patients included in the analyses of emotional triggering, income and depression did not differ from the remainder in age, gender or income distribution, the occurrence of emotional stress during the hazard period, ethnicity, employment status, cardiac history, GRACE scores or number of days spent in hospital. However, patients who failed to complete the 3-week interview were less likely to be married $(60.0 \%$ v. $72.2 \%, p=0.023)$ and were less likely to have a history of depression $(6.0 \% v$.
$30.8 \%, p<0.001)$ or depression in the year before ACS (0\% v. 6.1\%, $p<0.001)$.

Depression and anxiety data were obtained from 155 patients at 6 months and 155 patients at 12 months. Participation in the two phases did not overlap completely. Since associations with SES and emotional triggering were very similar at these two time points, they were combined to maximize numbers $(n=186)$. Patients who did not complete assessments at 6 or 12 months were younger on average $(57.55 \pm 12.5 v$. $60.94 \pm 10.7, p=0.023$ ) and more likely to be from an ethnic minority $(25.3 \%$ v. $13.8 \%, p=0.027)$. They did not differ from the remainder in any other characteristics.

\section{Results}

Approximately one-third of patients fell into each of the household income categories. Educational attainment was generally low, with $53.5 \%$ achieving basic educational qualifications, $30.7 \%$ secondary education and only $15.8 \%$ having college/university education. Education was positively associated with income $(r=0.22)$.

Table 1 summarizes patient characteristics according to household income categories. More female and unmarried patients had low household income $(p<0.001)$, lower income patients tended to be older $(p<0.001)$. Income category was not related to ethnicity, type of ACS or history of acute CHD (result not shown), but less affluent patients had worse GRACE risk scores $(p<0.001)$ and spent more days in hospital $(p=0.012)$. Ratings of life stress over the past 6 months did not differ across income categories. Overall, $22.9 \%$ of patients had a history of depressive illness, although only $12(4.3 \%)$ had been depressed in the 12 months before ACS onset. There was no difference between income groups in history of depression.

\section{Trigger analysis}

A total of 84 patients reported emotional stress during the 2-h hazard period ( 35 anger, 37 stress, and 29 depression or sadness), of whom 52 also reported emotional stress during the control period $24 \mathrm{~h}$ earlier. Altogether, 17 patients reported emotional stress during the control period only. The odds of onset of ACS following emotional stress were 1.88 (95\% CI 1.01-3.61).

A comparable analysis was conducted for physical exertion during the period before symptom onset. Of the 273 patients, 55 were physically active in the hazard period, 19 in the hazard and control periods and nine only in the control period. The odds of onset of ACS following physical exertion were 4.00 
Table 1. Study sample

\begin{tabular}{|c|c|c|c|c|}
\hline & $\begin{array}{l}\text { High income } \\
(>£ 40000) \\
(n=81)\end{array}$ & $\begin{array}{l}\text { Intermediate income } \\
(£ 20000-£ 40000) \\
(n=88)\end{array}$ & $\begin{array}{l}\text { Lower income } \\
(<£ 20000) \\
(n=110)\end{array}$ & $p$ \\
\hline Gender & & & & 0.001 \\
\hline Male & 78 & 83 & 76 & \\
\hline Female & 3 & 5 & 34 & \\
\hline Age (years) & $55.48 \pm 10.7$ & $58.49 \pm 10.8$ & $64.45 \pm 11.3$ & 0.001 \\
\hline Ethnicity (non-white) & $13(16.0 \%)$ & $12(13.6 \%)$ & $23(20.9 \%)$ & 0.33 \\
\hline Married & $70(86.4 \%)$ & $62(70.5 \%)$ & $56(50.9 \%)$ & 0.001 \\
\hline Vigorous activity/week & $1.62 \pm 1.95$ & $2.06 \pm 2.19$ & $2.30 \pm 2.46$ & 0.20 \\
\hline ST elevation MI & $68(84.0 \%)$ & $77(87.5 \%)$ & $100(90.9 \%)$ & 0.15 \\
\hline Grace risk score & $82.04 \pm 25.8$ & $89.01 \pm 25.9$ & $102.63 \pm 25.8$ & 0.001 \\
\hline Days in hospital & $4.40 \pm 2.0$ & $5.64 \pm 5.0$ & $6.13 \pm 4.2$ & 0.012 \\
\hline Life stress in past 6 months & $4.06 \pm 3.5$ & $3.36 \pm 3.1$ & $3.06 \pm 3.7$ & 0.14 \\
\hline History of clinical depression & $21(25.9 \%)$ & $14(15.9 \%)$ & $29(26.4 \%)$ & 0.82 \\
\hline Depression in the past 12 months & $4(4.9 \%)$ & $3(3.4 \%)$ & $5(4.5 \%)$ & 0.93 \\
\hline
\end{tabular}

MI, myocardial infarction.

(CI 1.89-9.44). We therefore replicated previous studies in showing that both emotional stress and physical exertion may acutely increase risk of ACS onset.

Emotional stress during the trigger period was more common among women patients (OR 2.23, CI $1.06-4.70, p=0.034)$, and was associated with younger age (OR 0.97, CI 0.95-0.99, $p=0.011$ ) and greater education (OR 1.56, CI 1.09-2.23, $p=0.015$ ). It was not related to household income. By contrast, physical exertion during the trigger period was associated with higher income (OR 1.92, CI 1.17-3.13, $p=0.009$ ), even after adjusting for age, gender and habitual physical activity. Neither emotional stress nor physical activity in the trigger period was associated with clinical indicators of disease including GRACE score, number of days in hospital, ACS type, previous MI or cardiac arrest. Life stress in the previous 6 months was associated with emotional stress $(r=0.30, p<0.001)$ but not physical exertion $(r=0.02, p=0.76)$ during the trigger period.

\section{Depression 3 weeks after ACS}

The mean BDI rating obtained during the home interviews conducted 3 weeks after ACS averaged $6.89 \pm 6.7$, while the mean HAMD score was $3.17 \pm 6.1$; $19.3 \%$ had BDI scores $\geqslant 10$. Analysis of variance of BDI ratings showed significant effects of emotional triggers $(F=7.10, p=0.008)$ and income $(F=8.06$, $p<0.001$ ), with greater depression in lower SES patients and in those who reported emotional stress during the hazard period. The emotional stress by income interaction was also significant $(F=6.88$, $p<0.001$ ) after adjustment for age, gender, ethnicity, (a)

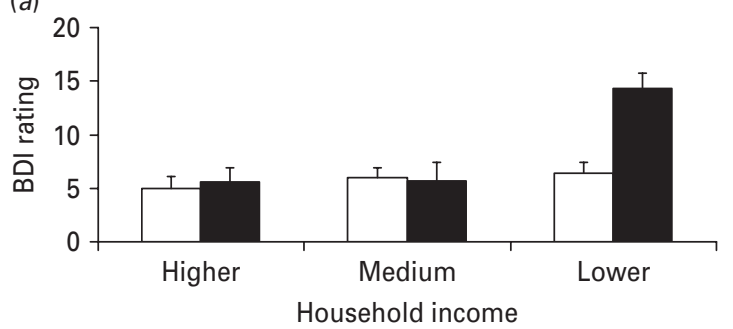

(b)

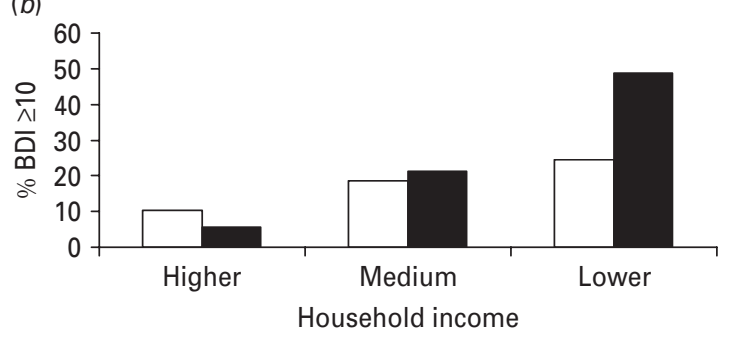

Fig. 1. Mean scores on the Beck Depression Inventory (BDI) (a) and the proportion of patients with BDI scores $\geqslant 10$. (b) 3 weeks after acute coronary syndrome (ACS) in patients with higher, medium and lower household incomes. Patients who experienced emotional stress in the hazard period before ACS onset ( $\boldsymbol{\square}$ ) and no emotional stress ( $\square$ ). Values are adjusted for age, gender, ethnicity, marital status, GRACE risk score, days in hospital and history of depression. Error bars are standard error of the mean.

marital status, GRACE score, number of days spent in hospital and history of depression. The results in Fig. 1 indicate that depression was substantially elevated in the lower income patients who experienced emotional stress during the trigger period, compared with all other categories. A similar pattern was recorded for 
HAMD scores, where the emotional trigger by income interaction was again significant $(F=6.62, p=0.002)$. HAMD scores were more than twice as high in the lower income/emotional trigger category (adjusted mean $9.59 \pm 9.55$ ) compared with all others (mean $2.65 \pm 5.22$ ). These differences were reinforced by analysis of the proportion of patients with BDI scores $\geqslant 10$ (Fig. 1). Just under $50 \%$ of lower income patients who experienced emotional stress during the trigger period had depression scores above threshold. A SES gradient was also apparent. In logistic regression on $\mathrm{BDI} \geqslant 10$, the interaction between income and emotional triggers was significant (adjusted OR 1.52, CI 1.16-2.00, $p=0.002$ ), controlling for covariates. Replacing history of depression with depression in the 12 months before ACS did not alter the results.

Analysis of HADS anxiety showed a similar pattern. Overall, anxiety scores averaged $4.80 \pm 4.3$ and $23.8 \%$ had values $\geqslant 8$. Analysis of covariance revealed significant effects for income $(F=6.55, p=0.002)$ and emotional triggering $(F=4.60, p=0.033)$, with higher anxiety in lower SES patients and those who experienced emotional stress in the 2 -h period before symptom onset, after controlling for covariates. The interaction was significant $(F=5.65, p=0.004)$, with adjusted anxiety scores averaging $8.63 \pm 6.2$ in the lower SES/emotional trigger category, compared with $4.42 \pm 3.8$ in all other categories. The proportion of patients in the lower SES/emotional trigger category with anxiety scores $\geqslant 8$ was $52.2 \%$, compared with $19.0 \%$ in the medium and $23.0 \%$ in the higher income categories. In logistic regression on HADS anxiety $\geqslant 8$, the interaction between income and emotional triggers was significant (adjusted OR 1.40, CI 1.08$1.81, p=0.010)$, controlling for covariates.

Physical exertion during the 2-h hazard period was unrelated to depression or anxiety 3 weeks after ACS, although the main effect for income remained significant $(F=4.06, p=0.019)$.

These analyses were repeated with educational attainment as the SES indicator. No consistent association with depression measured with the BDI or HAMD was observed and no significant interactions with emotional stress. Anxiety at 3 weeks was related to education $(F=2.87, p=0.040)$, with adjusted scores averaging 5.74, 4.89 and 3.72 in patients with basic, secondary, and college education respectively. But education did not interact with the occurrence of emotional stress during the hazard period.

\section{Emotional triggering, SES and well-being at 6 and 12 months}

The aggregate BDI rating at $6 / 12$ months was $8.19 \pm 8.5$, while HADS anxiety averaged $4.76 \pm 4.5$.
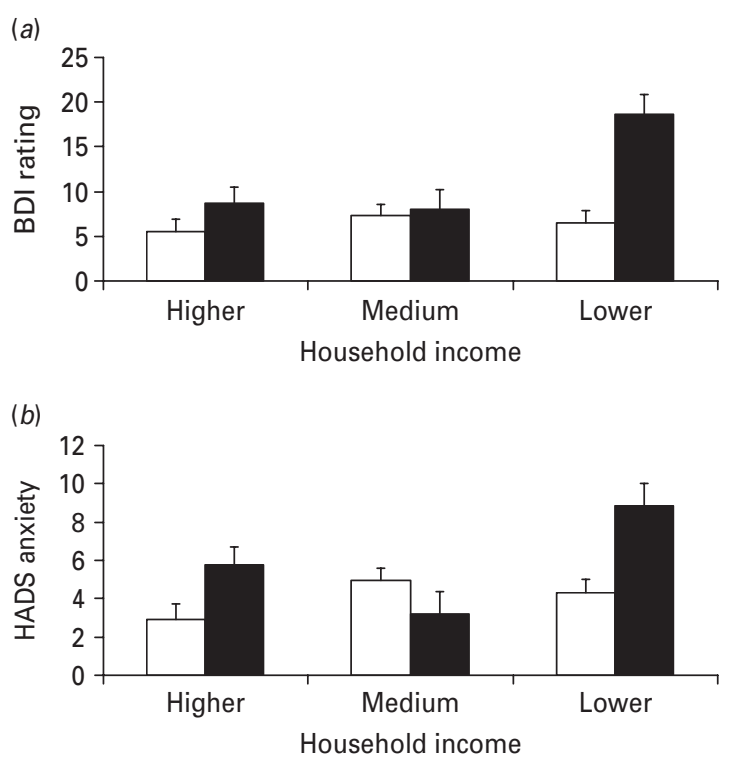

Fig. 2. Mean scores on (a) the Beck Depression Inventory and $(b)$ the Hospital Anxiety and Depression Scale (HADS) $6 / 12$ weeks after acute coronary syndrome (ACS) in patients with higher, medium and lower household incomes. Patients who experienced emotional stress in the hazard period before ACS onset ( $\square$ ) and no emotional stress $(\square)$. Values are adjusted for age, gender, ethnicity, marital status, GRACE risk score, days in hospital, history of depression and major adverse cardiac events. Error bars are standard error of the mean.

Depression ratings were significantly greater in lower SES participants $(F=4.74, p=0.010)$ and in those who experienced emotional stress during the trigger period $(F=14.82, p<0.001)$, controlling for age, gender, ethnicity, marital status, GRACE score, number of days spent in hospital, history of depression and major cardiac events in the intervening period. Additionally, the income/emotional trigger interaction was significant $(F=6.29, p=0.002)$ and is illustrated in Fig. 2. Patients in the lower SES/emotional trigger category had substantially higher BDI scores than all other categories (means $18.65 \pm 15.59$ v. $7.08 \pm 6.43$ ).

HADS anxiety ratings at $6 / 12$ months were also greater in lower income patients $(F=3.63, p=0.029)$ and those who experienced emotional stress $(F=6.52$, $p=0.012$ ) independently of covariates. As Fig. 2 illustrates, the interaction between income and emotional stress $(F=6.45, p=0.002)$ reflects higher anxiety in the lower income/emotional stress category, compared with all others.

These findings were replicated in separate analyses of 6 and 12 month emotional well-being (results not shown). Physical activity during the 2-h hazard period was not related to long-term emotional adaptation. Analyses with education as the indicator of SES 
showed no significant associations with longer-term emotional adaptation.

\section{Discussion}

The aim of this study was to discovery whether lower SES and emotional triggers influence the occurrence of depression and anxiety following ACS. We found that low household income but not education was related to elevated depression symptoms 3 weeks and 6 and 12 months after ACS independently of other demographic factors, clinical risk and history of depression. The impact of education was less consistent, with an association with anxiety 3 weeks after ACS but not later. The case-crossover analysis indicated that acute emotional stress and physical exertion were both associated with increased risk. Emotional stress but not physical exertion during the trigger period positively predicted symptoms of depression and anxiety 3 weeks after ACS and 6/12 months later. Most interestingly, there was an interaction between emotional triggers and low SES, such that depression and anxiety were particularly elevated in lower income patients who experienced emotional stress during the hazard period. These effects were substantial, with $48 \%$ of lower income/emotional stress patients having BDI scores $\geqslant 10$ and $50 \%$ having anxiety scores $\geqslant 8$ at 3 weeks.

The acute triggering effects were similar to those described in previous studies. For example, Mittleman et al. (1995) reported a relative risk of 2.3 (CI 1.7-3.2) of ACS following anger in the 2-h hazard period. An earlier study from our group showed an OR of 2.06 (CI 1.12-3.92) for anger and 2.50 (CI 1.05-6.56) for acute depression or sadness (Steptoe et al. 2006; Strike et al. $2006 b$ ). The association with physical exertion is also in line with previous studies, although we were not able to replicate the protective effect of regular physical exercise in this investigation (Willich et al. 1993).

The prevalence of elevated BDI scores 3 weeks after ACS was $19.3 \%$, within the range observed in other studies (Thombs et al. 2006). Previous studies have found inconsistent associations between SES and depression following ACS. Low education has been a significant predictor of depression in some studies (Grace et al. 2005; Parashar et al. 2006; Rutledge et al. 2006; Frasure-Smith et al. 2007; Carney et al. 2009) but not others (Frasure-Smith et al. 1993; Strik et al. 2004; Martens et al. 2008). Other markers of SES have been used less frequently, but Grace et al. (2005) reported that patients with lower family incomes were more likely to have elevated BDI scores in hospital. It should be emphasized that the lowest income category in the present study ( $<£ 20000$ per annum) was well above the poverty line in the UK (around $£ 6000$ and $£ 10400$ per annum for single and married individuals, respectively, at the time of the study), so patients in this category cannot be considered to have been in poverty.

The finding that household income predicted depression after ACS while education did not highlights the role of current socio-economic circumstances. Education is a marker of future socio-economic trajectories in younger people, predicts occupational opportunities and may equip people with problemsolving skills that are useful in adult life. But family income reflects access to material resources and this may be more relevant to emotional adjustment following ACS.

This study extends previous findings in showing an association between emotional stress immediately before symptom onset and depression and anxiety in the weeks following ACS. The interaction between lower income and emotional stress suggests that limited affluence may make cardiac patients particularly vulnerable to the longer-term emotional consequences of acute emotional stress at the time of ACS. The association may plausibly be a reflection of the general vulnerability of lower SES individuals to depressive symptoms after life stress (Harris et al. 1987; Kendler et al. 2002). Emotional stress during the trigger period was also associated with life stress over the 6 months before the ACS, indicating that it may occur in the context of chronic adversity. Interestingly, 52 of the 84 individuals reporting emotional stress during the 2-h hazard period also reported stress in the control period $24 \mathrm{~h}$ earlier, suggesting that much of the stress experienced in the prelude to ACS is sustained,

The mechanisms underlying these associations are not established. Lower SES individuals may lack material and social resources that allow them to cope effectively with serious cardiac disease. They may also be exposed to a greater extent to chronic stressors that augment the impact of heart disease. Lower SES individuals are less likely to make life-style behaviour modifications following ACS (Chan et al. 2008). Additionally, there are direct associations between lower SES and disturbed stress-related neuroendocrine and inflammatory processes that could contribute to risk of depression. For example, lower SES is associated with heightened inflammatory responses to acute stress and with cortisol dysregulation, both of which are implicated in the development of depression (Brydon et al. 2004; Kumari et al. 2010). Patients who have experienced acute emotional stress before ACS display heightened platelet activation following acute experimental stress (Strike et al. 2006a) and this may also be involved in depressive responses (Nemeroff \& Musselman, 2000). 
The strengths of this study include its prospective design, assessment of emotional triggers using a widely accepted interview protocol, measurement of depression with standard questionnaire and interview techniques and inclusion of comprehensive indicators of clinical cardiological risk. However, the conclusions that can be drawn from this study are limited by the setting of the investigation - a large hospital in an urban environment in the UK - and by attrition from the study sample. Participants in the study were younger and more likely to be male than those who were potentially eligible but did not take part, indicating that more fragile older women were less likely to be involved. Attrition before the 3-week interview was associated with marital status and history of depression; while at 6/12 months, ethnic minorities and younger patients were less likely to participate. The impact of these factors on the pattern of results is not easy to judge, since associations between lower SES, acute emotional stress and later depression and anxiety were independent of these characteristics.

There is currently debate about the merits of screening ACS patients for depression and identifying individuals who are at elevated risk for poor emotional adjustment (Thombs et al. 2008; Sowden et al. 2010). These findings indicate that attention might usefully be paid to patients' socio-economic circumstances and to their experiences shortly before hospital admission. Lower SES patients may be multiply disadvantaged, since they are not only more likely to develop depressive symptoms, but these symptoms are less likely to be recognized by clinicians (Amin et al. 2006) and they may also receive less active clinical intervention (Alter et al. 2004). In relation to emotional triggers, recommendations have been put forward for reducing exposure to triggering events and for taking precautions in high-risk situations (Tofler \& Muller, 2006; Steptoe \& Brydon, 2009), but little work has been done on suitable management of such patients after ACS. The results of this study suggest that emotional triggering may increase risk for depression in cardiac patients, so may contribute to better identification of vulnerable patients.

\section{Acknowledgements}

This research was supported by the British Heart Foundation (RG/05/006), Medical Research Council UK, and the Swiss National Science Foundation.

\section{Declaration of Interest}

None.

\section{References}

Alter DA, Chong A, Austin PC, Mustard C, Iron K, Williams JI, Morgan CD, Tu JV, Irvine J, Naylor CD $(2006 a)$. Socioeconomic status and mortality after acute myocardial infarction. Annals of Internal Medicine 144, 82-93.

Alter DA, Iron K, Austin PC, Naylor CD (2004). Socioeconomic status, service patterns, and perceptions of care among survivors of acute myocardial infarction in Canada. Journal of the American Medical Association 291, 1100-1107.

Alter DA, Venkatesh V, Chong A (2006b). Evaluating the performance of the Global Registry of Acute Coronary Events risk-adjustment index across socioeconomic strata among patients discharged from the hospital after acute myocardial infarction. American Heart Journal 151, 323-331.

Amin AA, Jones AM, Nugent K, Rumsfeld JS, Spertus JA (2006). The prevalence of unrecognized depression in patients with acute coronary syndrome. American Heart Journal 152, 928-934.

Beck AT, Steer RA, Garbin MG (1988). Psychometric properties of the Beck Depression inventory: twenty-five years of evaluation. Clinical Psychology Review 8, 77-100.

Bhattacharyya MR, Perkins-Porras L, Whitehead DL, Steptoe A (2007). Psychological and clinical predictors of return to work after acute coronary syndrome. European Heart Journal 28, 160-165.

Bhattacharyya MR, Perkins-Porras L, Wikman A, Steptoe A (2010). The long-term effects of acute triggers of acute coronary syndromes on adaptation and quality of life. International Journal of Cardiology 138, 246-252.

Brydon L, Edwards S, Mohamed-Ali V, Steptoe A (2004). Socioeconomic status and stress-induced increases in interleukin-6. Brain Behavior and Immunity 18, 281-290.

Carney RM, Freedland KE, Steinmeyer B, Blumenthal JA, de Jonge P, Davidson KW, Czajkowski SM, Jaffe AS (2009). History of depression and survival after acute myocardial infarction. Psychosomatic Medicine 71, 253-259.

Chan RH, Gordon NF, Chong A, Alter DA (2008). Influence of socioeconomic status on lifestyle behavior modifications among survivors of acute myocardial infarction. American Journal of Cardiology 102, 1583-1588.

de Jonge P, Rosmalen JG, Kema IP, Doornbos B, van Melle JP, Pouwer F, Kupper N (2010).

Psychophysiological biomarkers explaining the association between depression and prognosis in coronary artery patients: a critical review of the literature. Neuroscience and Biobehavioral Reviews 35, 84-90.

de Jonge P, van den Brink RH, Spijkerman TA, Ormel J (2006). Only incident depressive episodes after myocardial infarction are associated with new cardiovascular events. Journal of the American College of Cardiology 48, 2204-2208.

Dickens C, McGowan L, Percival C, Tomenson B, Cotter L, Heagerty A, Creed F (2008). New onset depression following myocardial infarction predicts cardiac mortality. Psychosomatic Medicine 70, 450-455.

Eagle KA, Lim MJ, Dabbous OH, Pieper KS, Goldberg RJ, van de Werf F, Goodman SG, Granger CB, Steg PG, Gore JM, Budaj A, Avezum A, Flather MD, 
Fox KA (2004). A validated prediction model for all forms of acute coronary syndrome: estimating the risk of 6-month postdischarge death in an international registry. Journal of the American Medical Association 291, 2727-2733.

Frasure-Smith N, Lesperance F, Irwin MR, Sauve C, Lesperance J, Theroux P (2007). Depression, C-reactive protein and two-year major adverse cardiac events in men after acute coronary syndromes. Biological Psychiatry 62, 302-308.

Frasure-Smith N, Lesperance F, Talajic M (1993). Depression following myocardial infarction. Impact on 6-month survival. Journal of the American Medical Association 270, 1819-1825.

Freedland KE, Skala JA, Carney RM, Raczynski JM, Taylor CB, Mendes de Leon CF, Ironson G, Youngblood ME, Krishnan KR, Veith RC (2002). The Depression Interview and Structured Hamilton (DISH) : rationale, development, characteristics, and clinical validity. Psychosomatic Medicine 64, 897-905.

Grace SL, Abbey SE, Kapral MK, Fang J, Nolan RP, Stewart DE (2005). Effect of depression on five-year mortality after an acute coronary syndrome. American Journal of Cardiology 96, 1179-1185.

Grippo AJ, Johnson AK (2009). Stress, depression and cardiovascular dysregulation: a review of neurobiological mechanisms and the integration of research from preclinical disease models. Stress 12, 1-21.

Harris T, Brown GW, Bifulco A (1987). Loss of parent in childhood and adult psychiatric disorder: the role of social class position and premarital pregnancy. Psychological Medicine 17, 163-183.

Ickovics JR, Viscoli CM, Horwitz RI (1997). Functional recovery after myocardial infarction in men: the independent effects of social class. Annals of Internal Medicine 127, 518-525.

Kendler KS, Gardner CO, Prescott CA (2002). Toward a comprehensive developmental model for major depression in women. American Journal of Psychiatry 159, 1133-1145.

Kloner RA (2006). Natural and unnatural triggers of myocardial infarction. Progress in Cardiovascular Diseases 48, 285-300.

Kronish IM, Rieckmann N, Schwartz JE, Schwartz DR, Davidson KW (2009). Is depression after an acute coronary syndrome simply a marker of known prognostic factors for mortality? Psychosomatic Medicine 71, 697-703.

Kumari M, Badrick E, Chandola T, Adler NE, Epel E, Seeman T, Kirschbaum C, Marmot MG (2010). Measures of social position and cortisol secretion in an aging population: findings from the Whitehall II study. Psychosomatic Medicine 72, 27-34.

Kurdyak PA, Gnam WH, Goering P, Chong A, Alter DA (2008). The relationship between depressive symptoms, health service consumption, and prognosis after acute myocardial infarction: a prospective cohort study. $B M C$ Health Services Research 8, 200.

Lichtman JH, Bigger Jr. JT, Blumenthal JA, Frasure-Smith N, Kaufmann PG, Lesperance F, Mark DB, Sheps DS, Taylor CB, Froelicher ES (2008). Depression and coronary heart disease: recommendations for screening, referral, and treatment: a science advisory from the American Heart Association Prevention Committee of the Council on Cardiovascular Nursing, Council on Clinical Cardiology, Council on Epidemiology and Prevention, and Interdisciplinary Council on Quality of Care and Outcomes Research: endorsed by the American Psychiatric Association. Circulation 118, 1768-1775.

Lorant V, Deliege D, Eaton W, Robert A, Philippot P, Ansseau M (2003). Socioeconomic inequalities in depression: a meta-analysis. American Journal of Epidemiology 157, 98-112.

Maclure M, Mittleman MA (2000). Should we use a case-crossover design? Annual Review of Public Health 21, 193-221.

Martens EJ, Smith OR, Winter J, Denollet J, Pedersen SS (2008). Cardiac history, prior depression and personality predict course of depressive symptoms after myocardial infarction. Psychological Medicine 38, 257-264.

Mittleman MA, Maclure M, Nachnani M, Sherwood JB, Muller JE (1997). Educational attainment, anger, and the risk of triggering myocardial infarction onset. The Determinants of Myocardial Infarction Onset Study Investigators. Archives of Internal Medicine 157, 769-775.

Mittleman MA, Maclure M, Sherwood JB, Mulry RP, Tofler GH, Jacobs SC, Friedman R, Benson H, Muller JE (1995). Triggering of acute myocardial infarction onset by episodes of anger. Circulation 92, 1720-1725.

Mittleman MA, Maclure M, Tofler GH, Sherwood JB, Goldberg RJ, Muller JE (1993). Triggering of acute myocardial infarction by heavy physical exertion. Protection against triggering by regular exertion. Determinants of Myocardial Infarction Onset Study Investigators. New England Journal of Medicine 329, 1677-1683.

Moller J, Theorell T, de Faire U, Ahlbom A, Hallqvist J (2005). Work related stressful life events and the risk of myocardial infarction. Case-control and case-crossover analyses within the Stockholm heart epidemiology programme (SHEEP). Journal of Epidemiology and Community Health 59, 23-30.

Nemeroff CB, Musselman DL (2000). Are platelets the link between depression and ischemic heart disease? American Heart Journal 140, 57-62.

Nicholson A, Kuper H, Hemingway H (2006). Depression as an aetiologic and prognostic factor in coronary heart disease: a meta-analysis of 6362 events among 146538 participants in 54 observational studies. European Heart Journal 27, 2763-2774.

Parashar S, Rumsfeld JS, Spertus JA, Reid KJ, Wenger NK, Krumholz HM, Amin A, Weintraub WS, Lichtman J, Dawood N, Vaccarino V (2006). Time course of depression and outcome of myocardial infarction. Archives of Internal Medicine 166, 2035-2043.

Parker GB, Hilton TM, Walsh WF, Owen CA, Heruc GA, Olley A, Brotchie H, Hadzi-Pavlovic D (2008). Timing is everything: the onset of depression and acute coronary syndrome outcome. Biological Psychiatry 64, 660-666.

Rutledge T, Reis SE, Olson MB, Owens J, Kelsey SF, Pepine CJ, Mankad S, Rogers WJ, Merz CN, Sopko G, Cornell CE, Sharaf B, Matthews KA, Vaccarino V (2006). Depression symptom severity and reported treatment 
history in the prediction of cardiac risk in women with suspected myocardial ischemia: The NHLBI-sponsored WISE study. Archives of General Psychiatry 63, 874-880.

Sowden G, Mastromauro CA, Januzzi JL, Fricchione GL, Huffman JC (2010). Detection of depression in cardiac inpatients: feasibility and results of systematic screening. American Heart Journal 159, 780-787.

Steptoe A, Brydon L (2009). Emotional triggering of cardiac events. Neuroscience and Biobehavioral Reviews 33, 63-70.

Steptoe A, Strike PC, Perkins-Porras L, McEwan JR, Whitehead DL (2006). Acute depressed mood as a trigger of acute coronary syndromes. Biological Psychiatry 60, 837-842.

Strik JJ, Lousberg R, Cheriex EC, Honig A (2004). One year cumulative incidence of depression following myocardial infarction and impact on cardiac outcome. Journal of Psychosomatic Research 56, 59-66.

Strike PC, Magid K, Whitehead DL, Brydon L, Bhattacharyya MR, Steptoe A (2006a). Pathophysiological processes underlying emotional triggering of acute cardiac events. Proceedings of the National Academy of Sciences USA 103, 4322-4327.

Strike PC, Perkins-Porras L, Whitehead DL, McEwan J, Steptoe A (2006b). Triggering of acute coronary syndromes by physical exertion and anger: clinical and sociodemographic characteristics. Heart 92, 1035-1040.

Strike PC, Steptoe A (2005). Behavioral and emotional triggers of acute coronary syndromes : a systematic review and critique. Psychosomatic Medicine 67, 179-186.
Thombs BD, Bass EB, Ford DE, Stewart KJ, Tsilidis KK, Patel U, Fauerbach JA, Bush DE, Ziegelstein RC (2006). Prevalence of depression in survivors of acute myocardial infarction. Journal of General Internal Medicine 21, 30-38.

Thombs BD, de Jonge P, Coyne JC, Whooley MA, Frasure-Smith N, Mitchell AJ, Zuidersma $M$, Eze-Nliam C, Lima BB, Smith CG, Soderlund K, Ziegelstein RC (2008). Depression screening and patient outcomes in cardiovascular care: a systematic review. Journal of the American Medical Association 300, 2161-2171.

Thygesen K, Alpert JS, White HD (2007). Universal definition of myocardial infarction. Journal of the American College of Cardiology 50, 2173-2195.

Tofler GH, Muller JE (2006). Triggering of acute cardiovascular disease and potential preventive strategies. Circulation 114, 1863-1872.

Warren-Gash C, Smeeth L, Hayward AC (2009). Influenza as a trigger for acute myocardial infarction or death from cardiovascular disease: a systematic review. Lancet Infectious Diseases 9, 601-610.

Willich SN, Lewis M, Lowel H, Arntz HR, Schubert F, Schroder R (1993). Physical exertion as a trigger of acute myocardial infarction. Triggers and Mechanisms of Myocardial Infarction Study Group. New England Journal of Medicine 329, 1684-1690.

Zigmond S, Snaith RB (1983). The Hospital Anxiety and Depression Scale. Acta Psychiatrica 86, 1-7. 